\title{
THE OPTIMAL LEBESGUE-RADON-NIKODYM INEQUALITY ${ }^{1}$
}

\author{
BY E. M. ALFSEN
}

Communicated by Edwin Hewitt, January 23, 1962

The aim of the present paper is to sketch some further developments of order-integration (cf. $[1 ; 2 ; 3]$ ), and in particular to point out how the absence of lattice distributivity introduces some new and interesting aspects of the Lebesgue-Radon-Nikodym Theorem. Details will be published elsewhere.

The formula $\mu(x, y)=v(y)-v(x)$ establishes a 1-1 correspondence between the set of valuations of a lattice $L$ (with identification modulo $v_{1} \sim v_{2} \Leftrightarrow v_{1}-v_{2}=$ const) and the set $L^{\prime}$ of projectivity invariant, additive interval-functions ("quotient-functions") on $L$. If $L$ is modular, then the equivalence classes of finite chains between $x$ and $y(x \leqq y)$ form a directed set in virtue of the Schreier-Ore Theorem. Hence we may define Riemann-Darboux integrals of projectivity invariant interval-functions in the natural way. The R. D. integral of $\mu$ is additive (whenever it exists) and will be denoted by $S_{\mu}$. Now the maximal directed vector subspace $L^{*}=\left(L^{\prime}\right)^{+}-\left(L^{\prime}\right)^{+}$of $L^{\prime}$ will consist of those $\mu \in L^{\prime}$ which are of bounded variation in the sense that

$$
S_{|\mu|}(x, y)=\sup _{x=x_{1} \leqq \ldots \leqq x_{n-y}} \sum_{i=1}^{n}\left|\mu\left(x_{i-1}, x_{i}\right)\right|<\infty ;
$$

for every interval $(x, y)$. Moreover, $L^{*}$ will be a conditionally complete vector lattice under the operations $\mu \vee \nu=S_{\mu \vee v}, \mu \wedge v=S_{\mu \wedge v}$. Decomposition of $\mu \in L^{*}$ in positive and negative parts yields the Jordan decomposition of $\mu$ (obtained by G. Birkhoff [4]).

The classical (Lebesgue-Vitali) definition of absolute continuity, $\nu \ll \mu$, for functions $\mu, \nu$ on $R$ can be directly transferred to the case in which $\mu, \nu$ belongs to the space $L^{*}$ of some modular lattice $L$. (The standard definition of $\nu \ll \mu$ for finitely additive measures $\mu, \nu$ on a Boolean ring is obtained from the general definition by reduction of the chain involved to a two-interval chain by application of the Boolean difference available in this particular case.) The concept of mutual singularity, $\mu \perp \nu$, can be defined for members of $L^{*}$ in an equally natural way. Let $\mathfrak{A}(\mu)$ denote the closed ideal ("famille com-

\footnotetext{
${ }^{1}$ Research supported in part by the National Science Foundation, U.S.A. (N.S.F.G18975).
} 
plète," "bande") generated by $\mu \in L^{*}$. Then it can be proved that:

$$
\nu \ll \mu \Leftrightarrow \nu \in \mathfrak{A}(\mu) ; \quad \nu \perp \mu \Leftrightarrow\|\nu\| \wedge\|\mu\|=0 \text {. }
$$

Hence F. Riesz' fundamental theorem on the decomposition of conditionally complete vector lattices into closed ideals [8; 6] yields a Lebesgue-decomposition in $L^{*}$ (proved by H. Bauer [5]).

Let $\mu$ be an arbitrary, but fixed positive member of the space $L^{*}$ of some modular lattice $L$. Then $\mu$ is a (weak) order unit of $\mathfrak{A}(\mu)$. Assume henceforth that $L$ has a least element $\phi$ and a greatest element $e$. Then $\mathfrak{A}(\mu)$ is an abstract $L$-space in the sense of Kakutani [7] under the norm $N(\nu)=\|\nu\|(\phi, e)=S_{|\nu|}(\phi, e)$. Hence $\mathfrak{A}(\mu)$ is isomorphic to $L^{1}(S, \mathcal{F}, m)$ where $(S, \mathfrak{F}, m)$ is an essentially unique, totally finite measure space whose measure algebra $\tilde{F}$ (i.e. $\mathcal{F}$ modulo null sets) is canonically isomorphic to the complete Boolean algebra $B$ of closed subideals of $\mathfrak{A}(\mu)$. We call $(S, \mathfrak{F}, m)$ the representation space of $\mathfrak{A}(\mu)$, and we call the representative function $f_{\nu} \in L^{1}(S, \mathcal{F}, m)$ of $\nu \in \mathfrak{Y}(\mu)$ the density function of $\nu$ relative to $\mu$.

For every $x \in L$ the annihilators

$$
\begin{aligned}
& \mathfrak{W}_{x}=\{\nu \mid \nu \in \mathfrak{A}(\mu), \nu \equiv 0 \text { on }[x, e]\}, \\
& \mathfrak{W}_{x}^{\prime}=\{\nu \mid \nu \in \mathfrak{U}(\mu), \nu \equiv 0 \text { on }[\phi, x]\}
\end{aligned}
$$

are closed subideals of $\mathfrak{U}(\mu), \mathfrak{W}_{x} \cap \mathfrak{S}_{x}^{\prime}=(0)$, and $x \rightarrow \mathfrak{W}_{x}, x \rightarrow \mathbb{\Omega}_{x}=L^{*} \ominus \mathfrak{S}_{x}^{\prime}$ are "meet"-preserving and "join"-preserving mappings of $L$ into $B$, respectively. Let $\pi^{*}$ be the "lifting" of the mapping $y \rightarrow x \wedge y$ from $L$ to the set of (not necessarily projectivity invariant) interval functions. For every $\nu \in\left(L^{*}\right)^{+}$the components $P_{x} \nu, Q_{x} \nu$ of $\nu$ into $\mathfrak{S}_{x}, \boldsymbol{\Omega}_{x}$ are the greatest and smallest projectivity invariant interval-functions, respectively, such that:

$$
P_{x \nu} \leqq \pi^{*} \nu \leqq Q_{x} \nu
$$

From this one can conclude:

(i) $P_{x} \nu$ is the greatest member of $L^{*}$ for which $0 \leqq P_{x} \nu \leqq \nu$ and $P_{x} \nu \equiv 0$ on $[x, e]$. On the other hand, $Q_{x} \nu$ is the smallest member of $L^{*}$ for which $0 \leqq Q_{x} \leqq \leqq$ and $Q_{x} \nu \equiv \nu$ on $[\phi, x]$.

(ii) If $x$ belongs to the distributive center $Z$ of $L$ (i.e. if $x$ forms distributive triples with any two elements of $L$ ), then $y \rightarrow x \wedge y$ is lattice preserving, hence $\pi^{*} \nu$ is projectivity invariant, and so we have equality signs in (3). Thus $x \in Z$ implies $\mathfrak{S}_{x}=\mathfrak{\Omega}_{x}$.

We denote the members of $\tilde{F}$ corresponding canonically to $\mathfrak{W}_{x}, \mathfrak{\Omega}_{x}$, by $H_{x}, K_{x}$, and recall that the mappings $x \rightarrow H_{x}, x \rightarrow K_{x}$ will be "meet"preserving and "join"-preserving, respectively, and that $H_{x} \subset K_{x}$, 
with $H_{x}=K_{x}$ whenever $x \in Z$. Now, we consider an arbitrary $\nu \in \mathfrak{A}(\mu)^{+}$ corresponding to a normalized valuation $v$ (i.e. $v(\phi)=0$ ), and by the correspondence $v \leftrightarrow \nu$ we may write $f_{v}$ instead of $f_{v}$. By means of the explicit construction of the Kakutani representation and the possibility of a spectral resolution in $\mathfrak{A}(\mu)$, we can prove the following general Lebesgue-Radon-Nikodym inequality:

$$
\int_{\boldsymbol{H}_{\boldsymbol{x}}} f_{v} d m \leqq v(x) \leqq \int_{\boldsymbol{K}_{\boldsymbol{x}}} f_{v} d m
$$

This result is in fact optimal in the sense that if

$$
\int_{A} f_{v} d m \leqq v(x) \leqq \int_{B} f_{v} d m_{H}
$$

for all $\nu \in \mathfrak{A}(\mu)$, then $A \subset H_{x} \subset K_{x} \subset B$. (Passage from the actual members of $\tilde{F}$ to representatives belonging to $\mathscr{F}$, will of course transfer the last relations to inclusions modulo null-sets.) To verify this assertion, we consider the component $\nu$ of $\mu$ into the closed subideal $\mathfrak{A}$ corresponding (canonically) to $A$. Then $f_{\nu}=\chi_{A}$, and so $\nu(\phi, e)=\int f_{\nu} d m$ $=m(A)$. By (5) we also have $\nu(\phi, x) \geqq \int_{A} f_{\nu} d m=m(A)$, and hence $\nu(x, e)=\nu(\phi, e)-\nu(\phi, x) \leqq 0$. By the positivity of $\nu$ this entails $\nu(x, e)$ $=0$, and in fact $\nu \equiv 0$ on $[x, e]$. In virtue of (i) we have $\nu \leqq P_{x} \mu$, and so $\mathfrak{A} \subset \mathfrak{S}_{x}$ proving that $A \subset H_{x}$. Similarly we can prove $K_{x} \subset B$.

It follows from (ii) that the distributive center $Z$ of $L$ is mapped homomorphically into $\tilde{\mathfrak{F}}$ by $x \rightarrow H_{x}\left(=K_{x}\right)$, and that:

$$
v(x)=\int_{H_{x}} f_{v} d m
$$

for $\nu \in \mathfrak{A}(\mu)$ and $x \in Z$. (Actually (6) remains valid even if we only require the equivalence class $\bar{x}$ of $x$ modulo $v$ to belong to the distributive center of $Z /[v]$.) In particular, we shall have an exact Lebesgue-Radon-Nikodym Theorem (6) on distributive lattices.

If $L$ is taken to be a Boolean algebra, then $v$ becomes a finitely additive measure (recall $v(\phi)=0$ ), and the mapping $x \rightarrow H_{x}$ will be a representation (but not necessarily a $\sigma$-representation) of $L$ into B. If $L$ is taken to be the Boolean algebra $\mathcal{G}$ of some totally finite measure space $(T, \mathcal{G}, p)$ and $v=p$, then the $\sigma$-continuity of $v$ will imply that $x \rightarrow \mathscr{S}_{x}\left(=\mathfrak{\Omega}_{x}\right)$ is a $\sigma$-representation, and hence so is $x \rightarrow H_{x}\left(=K_{x}\right)$. From this one can conclude that $(T, \mathcal{Q}, p)$ is one of the possible realizations of the (essentially unique) representation space. Thus we obtain the standard Lebesgue-Radon-Nikodym Theorem in this case. 


\section{REFERENCES}

1. E. M. Alfsen, On a general theory of integration based on order, Math. Scand. 6 (1958), 67-79.

2. - A remark concerning the article: "On a general theory of integration based on order," Math. Scand. 7 (1959), 106-108.

3. D. A. Kappos und A. Mallios, Der Integralbegriff in o-topologischen Verbänden, Bull. Soc. Math. Grèce, N.S. 1, Fasc. 1 (1960), 98-105.

4. G. Birkhoff, Lattice theory, Amer. Math. Soc. Coll. Publ. Vol. 25, Amer. Math. Soc., New York, 1948.

5. H. Bauer, Reguläre und singuläre Abbildungen eines Verbandes in einen vollständigen Vektorverband, welche der Funktionalgleichung $f(x \vee y)+f(x \wedge y)=f(x)+f(y)$, genügen, J. Reine Angew. Math. 194 (1955), 141-179.

6. N. Bourbaki, Intégration, Actualités Sci. Ind., no. 1175, Hermann, Paris, 1952.

7. S. Kakutani, Concrete representation of abstract $(L)$-spaces and the mean ergodic theorem, Ann. of Math. (2) 42 (1941), 523-537.

8. F. Riesz, Sur quelque notions fondamentales dans la théorie générale des opérations linéaires, Ann. of Math. (2) 41 (1940), 174-206.

UNIVERSITY OF WASHINGTON AND UNIVERSITY OF OSLO 